\title{
Eventos adversos pós-vacinais papilomavírus humano em adolescentes
}

\author{
Post-immunization adverse human papilomavirus events in teenagers \\ Ventos adversos post-vaccinarios del papiloma humano en adolescentes
}

\begin{abstract}
RESUMO
Avaliar a ocorrência dos eventos adversos pós-vacinais em adolescentes relacionados à vacina quadrivalente Papilomavírus Humano 6, 11, 16 e 18 (recombinante) através do Sistema de Informações do Programa Nacional de Imunizações Eventos Adversos Pós-Vacinação da Coordenadoria de Saúde da Área Programática 5.1 procedentes das Unidades Básicas de Saúde. A amostra foi composta por adolescentes entre 9 a 17 anos de idade. Estudo retrospectivo, de caráter exploratório, descritivo e quantitativo no período de 01 de novembro de 2016 até 01 de junho de 2019. Analisadas no estudo 22 fichas de notificações, sendo 19 de eventos adversos pós-vacinais e 3 Erros de Imunização (EI). Os eventos adversos nos adolescentes estão relacionados a ansiedade e medo frente a vacinação, ocorridos na administração da primeira dose da vacina HPV, predominante no sexo masculino, ocorrendo nos primeiros 30 minutos após administração do imunobiológico. Nesta pesquisa, os eventos adversos com maiores frequências, foram classificados como não graves, com evolução para cura sem sequelas, relacionados à manifestações como a palidez, hipotensão, desmaio e síncope.
\end{abstract}

DESCRITORES: Papilomavírus Humano (HPV); Saúde do Adolescente; Imunização; Efeitos Adversos.

\section{ABSTRACT}

To assess the occurrence of post-vaccine adverse events in adolescents related to the human papillomavirus quadrivalent vaccine 6, 11, 16 and 18 (recombinant) through the National Immunization Program Information System Post-Vaccination Adverse Events of the Program Area Health Coordination 5.1 from the Basic Health Units. The sample consisted of adolescents between 9 and 17 years old. Retrospective, exploratory, descriptive, and quantitative study from November 1, 2016 to June 1, 2019. The study analyzed 22 reporting forms, 19 of adverse events following vaccination and 3 Immunization Errors (IS). Adverse events in adolescents are related to anxiety and fear of vaccination occurring in the administration of the first dose of the predominant male HPV vaccine occurring within the first 30 minutes after immunobiological administration. In this research, the most frequent adverse events were classified as non-serious, with evolution to cure without sequelae, related to manifestations such as pallor, hypotension, fainting and syncope.

DESCRIPTORS: Human Papillomavirus (HPV); Adolescent Health; Immunization; Adverse effects.

\section{RESUMEN}

Evaluar la aparición de eventos adversos posteriores a la vacuna en adolescentes relacionados con la vacuna cuadrivalente al virus del papiloma humano 6, 11, 16 y 18 (recombinante) a través del Sistema Nacional de Inmunización del Sistema de Información de Eventos Adversos Post-Vacunación del Área del Programa Coordinación de Salud 5.1 de las Unidades Básicas de Salud. La muestra estuvo conformada por adolescentes entre 9 y 17 años. Estudio retrospectivo, exploratorio, descriptivo y cuantitativo del 1 de noviembre de 2016 al 1 de junio de 2019. El estudio analizó 22 formularios de informe, 19 de eventos adversos después de la vacunación y 3 errores de inmunización (IS). Los eventos adversos en los adolescentes están relacionados con la ansiedad y el miedo a que ocurra la vacunación en la administración de la primera dosis de la vacuna contra el VPH masculina predominante dentro de los primeros 30 minutos después de la administración inmunobiológica. En esta investigación, los eventos adversos más frecuentes se clasificaron como no graves, con evolución para curar sin secuelas, relacionados con manifestaciones como palidez, hipotensión, desmayos y síncope.

DESCRIPTORES: Virus del Papiloma Humano (VPH); Salud del Adolescente; Inmunización; Efectos adversos.

RECEBIDO EM: 11/01/2020 APROVADO EM: 12/01/2020

\section{Maria Regina Bernardo da Silva}

Mestre em Saúde da Família e Comunidade. MBA em gestão em Saúde. Docente da Universidade Castelo Branco e UNIBCE/RJ. https://orcid.org/0000-0002-3620-3091 


\section{Aline Silvano Frutuoso Conceição}

Enfermeira pela Universidade Castelo Branco. https://orcid.org/0000-0001-8464-8493

\section{Douglas Magno da Silva}

Enfermeiro pela Universidade Castelo Branco. https://orcid.org/0000-0002-5633-4822

\section{Angela Dias de Araujo Ramado}

Enfermeira pela Universidade Castelo Branco. https://orcid.org/0000-0002-8222-351X

\section{Jane Gregório de Andrade}

Enfermeira pela Universidade Castelo Branco. https://orcid.org/0000-0002-5288-9580

\section{Tamires Lucena Reis}

Enfermeira pela Universidade Castelo Branco. https://orcid.org/0000-0003-2279-0368

\section{Renata Salles de Souza}

Enfermeira pela Universidade Castelo Branco. https://orcid.org/0000-0001-5117-157X

\section{INTRODUÇÃO}

0 Vírus do Papiloma Humano é uma infecção sexualmente transmissível, são mais de 200 variações desse tipo de vírus que atacam, especialmente, as mucosas oral, genital ou anal, tanto o sexo masculino como o feminino, porém, na sua maioria, está associada a lesões benignas, como as verrugas, que podem ser clinicamente removidas ${ }^{(1)}$.

Os preservativos (camisinha masculina e feminina) previnem a maioria das Infecções Sexualmente Transmissíveis (IST), no caso do HPV, eles não impedem totalmente a infecção pelo vírus. A transmissão pode ocorrer mesmo sem a penetração vaginal ou anal, e as lesões podem estar presentes em áreas não protegidas pelos preservativos, como: vulva, região pubiana, perineal e perianal ou bolsa testicular ${ }^{(2)}$.

De acordo com o Ministério da Saú$\operatorname{de}^{(1)}$ no Brasil, há predominância na circulação de quatro subtipos que atingem tanto homens quanto mulheres e, segundo a literatura científica, 12 subtipos de HPV estão associados aos cânceres do colo do útero, de pênis, de orofaringe e, até mesmo, de câncer reto-anal.

Conforme publicação do $\operatorname{INCA}^{(3)} \mathrm{o}$ câncer do colo do útero é o quarto tipo de câncer mais comum de morte entre as mulheres, excetuando-se os casos de pele não melanoma, com aproximadamente 530 mil casos novos por ano no mundo e sendo responsável por 265 mil óbitos anuais. Está associado à infecção persistente por subtipos oncogênicos do vírus HPV (Papilomavírus Humano), especialmente o HPV-16 e o HPV-18, responsáveis por cerca de $70 \%$ dos cânceres cervicais.

As vacinas são produtos biológicos com excelente segurança e as ocorrências de eventos adversos relacionados aos imunobiológicos deve ser de notificação imediata $^{(4)}$. Criado em 1973, o Programa Nacional de Imunizações (PNI) é responsável pela organização da política nacional de vacinação da população brasileira. A vacinação contribui para a redução da morbidade e mortalidade por doenças transmissíveis no Brasil( ${ }^{(5)}$.

O Programa Nacional de Imunizações (PNI) vem oferecendo, de forma ordenada, nas salas de imunizações das Unidades de Saúde, vacinas contra doenças transmissíveis através de realização de campanhas, favorecendo para o controle e erradicação de doenças. Nos casos de hipersensibilidade às vacinas da rotina ou em algumas situações de imunodepressão, nestas situações particulares, as vacinas são realizadas nos Centros de Referência de Imunobiológicos Especiais - CRIE ${ }^{(6)}$.

Considera-se um evento adverso pós vacinal (EAPV), segundo o Ministério da Saúde ${ }^{(4)}$, qualquer ocorrência médica indesejada após imunização e que, não impreterivelmente, possua uma associação causal com o uso de uma vacina ou outro imunobiológico (imunoglobulinas e soros heterólogos). A maioria das ocorrências dos eventos adversos são manifestações locais e sistêmicos leves. A intensidade dos mesmos pode variar desde efeitos leves e esperados, como as manifestações locais, até os eventos moderados, graves ou casos raros. classificados como inesperados ${ }^{(7)}$.

Os componentes do imunobiológico, $o$ vacinado e o processo de vacinação são fatores que podem estar relacionados a um EAPV, no entanto, quando a prática profissional, as normas e técnicas não forem cumpridas, resultando, ou não, em um evento adverso, este, se caracteriza com um Erro de Imunização (EI). Sendo qualquer evento evitável que pode causar ou levar a um uso inapropriado ou causar dano a um paciente ${ }^{(8)}$.

Segundo Ministério da Saúde ${ }^{(1)}$, no ano de 2014, iniciou-se a introdução da vacina quadrivalente recombinante contra HPV no Programa Nacional de Imunizações (PNI), contemplando meninas entre $11 \mathrm{e}$ 13 anos com objetivo principal de imunização das mulheres contra o câncer do colo do útero. Estudos evidenciam a malignidade associada ao Papilomavírus Humano em mulheres e não maligno nos homens $s^{(9)}$.

O Ministério da Saúde do Brasil e outros países reavaliaram suas análises de custo/efetividade da inclusão dos meninos nas campanhas de imunização e, em 2017, ocorreu a ampliação da faixa etária para a vacinação entre os meninos a partir dos 


\section{artigo}

Bernado da Silva, M.R.; Conceição, A.S.F.; Magno da Silva, D.; Ramado, A.D.A.; Andrade, J.G.; Reis T.L.; Souza, R.S.;

Eventos adversos pós-vacinais papilomavírus humano em adolescentes

11 anos até os 15 anos incompletos e as meninas a partir dos 9 anos até os 15 anos incompletos. A vacina deve ser tomada em duas doses, com intervalo mínimo de seis meses e máximo de um ano entre elas. Os indivíduos de 9 a 26 anos de ambos os sexos convivendo com HIV/Aids, pacientes oncológicos em quimioterapia e/ ou radioterapia; transplantados de órgãos sólidos ou de medula óssea., também são comtemplados com o imunobiológico ${ }^{(1)}$.

A Sociedade Brasileira de Pediatria (SBP), assim como a Sociedade Brasileira de Imunizações (SBIm) e a Federação Brasileira das Associações de Ginecologia e Obstetrícia (FEBRASGO), recomendam a vacinação de meninas e mulheres de 9 a 45 anos de idade e meninos e jovens de 9 a 26 anos. Homens e mulheres em idades fora da faixa de licenciamento também podem ser beneficiados com a vacinação, de acordo com critério médico ${ }^{(9)}$.

Por se tratar de uma vacina incorporada recentemente ao calendário vacinal do adolescente pelo Ministério da Saúde no Brasil e com relatos de eventos adversos, que desqualificam essa forma de prevenção, tal fato, motivou o presente estudo. A pesquisa justifica-se pela necessidade de avaliar os casos de eventos adversos pós vacinais, se estão associados à substância vacinal ou são casos de eventos isolados relacionados ao próprio indivíduo. Sendo relevante o estudo do perfil de segurança para o grupo em que a vacina é disponibilizada, visto que, é uma população de pouca adesão aos programas de imunizações e sua utilização como forma profilática evita doenças infecciosas e reduz a incidência de câncer do colo do útero ${ }^{(4)}$.

A vacinação contra o HPV é um importante benefício vantajoso e favorável para sociedade, como proteção coletiva. No espaço acadêmico, o estudo fornece dados e orientações às futuras pesquisas associadas aos eventos adversos pós vacinais do Papilomavírus Humano. Enriquecendo assim a formação de novos profissionais, seja nos níveis técni$\cos$, de graduação ou de pós-graduação ${ }^{(4)}$.

E o objetivo geral é avaliar a ocorrência dos eventos adversos pós-vacinais em adolescentes relacionados à vacina quadri- valente Papilomavírus Humano 6, 11, 16 e 18 (recombinante) através do Sistema de Informações do Programa Nacional de Imunizações Eventos Adversos Pós-Vacinação da Coordenadoria de Saúde da Área Programática 5.1.

\section{METODOLOGIA}

A presente pesquisa é um estudo retrospectivo, de caráter exploratório, descritivo e quantitativo. Segundo estudo ${ }^{(10)}$, a abordagem exploratória é desenvolvida no sentido de proporcionar uma visão geral acerca do tema estudado, consiste no aprofundamento do tema, proporcionando maiores informações e contribuindo para o esclarecimento de questôes abordadas no assunto.

A pesquisa descritiva tem como principal objetivo, descrever características de determinada população ou fenômeno, estabelecendo relações entre as variáveis, preocupando-se em observar os fatos, registrando, analisando, classificando e interpretando sem que exista a interferência por parte do pesquisador nos resultados obtidos ${ }^{(11)}$.

A investigação exploratória é habitualmente, o primeiro passo para quem não conhece suficientemente o campo que pretende aludir e sendo realizada em área na qual há pouco conhecimento acumulado $\mathrm{e}$ sistematizado ${ }^{(12)}$, e no estudo retrospectivo estudam-se casos e controles, se conhece o efeito e se busca a causa ${ }^{(13)}$.

$\mathrm{Na}$ pesquisa quantitativa, a determinação da composição e do tamanho da amostra é um processo no qual a estatística tornou-se o meio principal. As respostas de alguns problemas podem ser deduzidas para o todo, então, a amostra deve ser muito bem definida; caso contrário, podem surgir problemas ao se utilizar a solução para o todo ${ }^{(14)}$.

Elaborado a partir de um levantamento de dados do Sistema de Informações do Programa Nacional de Imunizações módulo Sistema de Informações Eventos Adversos Pós-Vacinação (SI-PNI/SI-EAPV), procedentes das Unidades Básicas de Saúde (UBS) sendo estas, referentes à Coordenadoria de Saúde da Área Programática
(CAP) 5.1, situada em Jardim Sulacap, no município do Rio de Janeiro. Foram incluídos registros de notificações dos eventos adversos pós-vacinais da vacina quadrivalente Papilomavírus Humano 6, 11, 16 e 18 recombinante em adolescentes.

A escolha do Sistema de Informações do Programa Nacional de Imunizações módulo Sistema de Informações Eventos Adversos Pós-Vacinação (SI-PNI/SI-EAPV) para o estudo foi pertinente pela compreensibilidade de acompanhar os eventos adversos, com vertiginosa identificação dos casos, classificações e condutas ocorridas na Área Programática 5.1.

Foram fontes as fichas de notificações com a faixa etária entre 09 a 17 anos de idade, ambos os sexos, com queixas de EAPV, referentes ao imunobiológico quadrivalente do Papilomavírus Humano, no período de 01 de novembro de 2016 até 01 de junho de 2019, realizando um levantamento de todos os eventos adversos pós-vacinais.

Foram analisadas no estudo as variáveis da idade dos adolescentes, sexo, cor, dose da vacina, tipo de eventos adversos, como manifestações locais, clínicas sistêmicas (pele/mucosas, cardiovasculares, respiratórias, neurológicas e gastrointestinais) e outras manifestações. Além do intervalo de início, vacinas administradas simultaneamente, parecer da Coordenação do Programa de Imunizações (CPI) e se há relação direta desses eventos com a vacina, baseadas nas notificações realizadas pelas Unidades Básicas de Saúde da Coordenadoria de saúde da Área Programática 5.1.

Os dados foram relatados a partir dos resultados das variáveis, submetidos à análise de estatística de forma simples, apresentados através de tabelas no Microsoft Excel que facilitaram a interpretação das informações. Por se tratar de coleta de dados envolvendo seres humanos, a pesquisa foi submetida ao Comitê de Ética em Pesquisa (CEP) de acordo com a Resolução n. ${ }^{\circ}$ 466/12 e a Resolução n. ${ }^{\circ}$ 510/16, com aprovação do Comitê de Ética da Secretaria Municipal de Saúde do Rio de Janeiro (SMS /RJ) parecer n. ${ }^{\circ}$ 3.593.283. 


\section{RESULTADOS E DISCUSSÃO}

Foram analisadas no estudo 22 fichas de notificações, sendo 19 de eventos adversos pós-vacinais e 3 Erros de Imunização (EI), preservando a identidade dos indivíduos, referentes ao imunobiológico do Papillomavírus Humano quadrivalente 6, 11, 16 e 18 (recombinante).

Entre 2016 e 2019 nos 22 casos notificados como EAPV na plataforma SIPNI/ SIEAPV, as maiores frequências de notificações ocorreram em 2016 e 2017, com 10 a cada ano, e 2 em 2018. No ano de 2019 não há registro de notificações no Sistema.

O profissional ao realizar a inserção da notificação no Sistema de Informações do Programa Nacional de Imunizações módulo Sistema de Informações Eventos Adversos Pós-Vacinação, no campo de manifestações, o paciente cadastrado que apresente algum sinal e sintoma que não seja descrito, este, deve ser especificado e classificado como outras manifestações.

A amostra foi composta por adolescentes, meninos e meninas entre 9 a 18 anos de idade, 14 do sexo masculino e 8 do sexo feminino. Dentre os 22 casos, 03 foram

Tabela 1. Descrição das variáveis idade, sexo, cor, dose da vacina e tempo de início dos casos relacionados aos eventos adversos pós-vacinais relacionados à vacina contra o Papillomavírus Humano quadrivalente 6, 11, 16 e 18 (recombinante). Rio de Janeiro, RJ, Brasil, 2016-2019.

Variáveis

Quantidade

Erro de Imunização

Idade

$$
9
$$

14

17 e 18

Sexo

Feminino

Masculino

Dose da vacina

$$
\begin{aligned}
& 1^{a} \text { dose } \\
& 2^{a} \text { dose }
\end{aligned}
$$

Cor

Amarela 2

Branca 5

Parda 7

Não informada 5

Tempo de início do efeito adverso

Não informado

$\begin{array}{cc}30 \text { minutos } & \mathbf{4} \\ 3 \text { horas } & 1 \\ 4 \text { horas } & 1 \\ 6 \text { horas } & 1 \\ \text { Não informado } & 12\end{array}$

5

notificados como Erro de Imunização (EI), com idades de 9, 10, 17 e 18 anos, 02 do sexo masculino e 01 do feminino.

Realizada busca no Sistema de Informações de Saúde do departamento de informática do SUS (TABNET-DATASUS) para analisar a predominância dos eventos adversos em adolescentes do sexo masculino. O DATASUS proporciona informações que podem ser úteis para análises objetivas da situação sanitária, elaboração de programas de ações de saúde e tomadas de decisão baseadas em evidências. Os resultados demonstram o aumento do número de adolescentes do sexo masculino vacinados na faixa etária analisada.

Analisando os resultados através da fonte TABNET-DATASUS, em relação ao número maior de reações adversas nos adolescentes do sexo masculino, o objetivo primário da vacinação contra o HPV em 2014, era a proteção das mulheres contra o câncer do colo do útero e, a partir de 2017, estendeu-se para os homens. Devido à inclusão dos meninos no Programa de Imunizações para oferta da vacina do HPV, o número de adolescentes do sexo masculino vacinados aumentou, tornou-se mais evidente a probabilidade de eventos adversos.

No que se refere à dose da vacina, todos os eventos adversos ocorridos foram relacionados à primeira dose do esquema vacinal do Papilomavírus Humano quadrivalente. As notificações inseridas na plataforma como EI apresentam menina com 9 anos de idade que foi realizado D1 em 08/11/2016 e D2 em 29/03/2017, ou seja, administração da vacina com intervalo de aproximadamente 3 meses e 15 dias entre as doses, sendo o preconizado 6 meses da D1 para D2. Outro caso, o adolescente do sexo masculino com 10 anos estava fora das faixas etárias recomendadas pelo Programa Nacional de Imunizações (PNI), na época meninas 09 a 14 anos e meninos de 12 a 13 anos. Em 01 adolescente ocorreram dois erros, na $1^{\text {a }}$ com 17 anos e na $2^{\text {a }}$ dose com 18 anos, que foram administradas também fora da faixa etária preconizada na época para os meninos.

Em relação à cor dos adolescentes, em 12 casos não foram informados a 


\section{artigo}

Bernado da Silva, M.R.; Conceição, A.S.F.; Magno da Silva, D.; Ramado, A.D.A.; Andrade, J.G.; Reis T.L.; Souza, R.S.

Eventos adversos pós-vacinais papilomavirus humano em adolescentes

cor, contudo, nos informados a predominância é a cor parda. Não há relatos na literatura associando os eventos adversos com a cor da pele do indivíduo, no entanto, a Pesquisa Nacional por Amostra de Domicílios Contínua (Pnad Contínua) 2016, divulgada pelo Instituto Brasileiro de Geografia e Estatística (IBGE), revela que, a maioria da população brasileira residente é parda, são

Tabela 2. Descrição por Área Programática 5.1, classificação, descartados, encerrados e Erro de Imunização dos casos relacionados aos eventos adversos pós-vacinais notificados da vacina contra o Papilomavírus Humano quadrivalente 6, 11, 16 e 18 (recombinante). Rio de Janeiro, RJ, Brasil, 2016-2019.

$\begin{array}{cccccccc}\text { AP } & \begin{array}{c}\text { Não } \\ \text { grave }\end{array} & \text { Grave } & \begin{array}{c}\text { Mudança de } \\ \text { classificação }\end{array} & \text { Total } & \text { Descartados } & \text { Encerramento } & \begin{array}{c}\text { Erro de } \\ \text { Imunização }\end{array} \\ 5.1 & 18 & 1 & 1 & 19 & 0 & 22 & 3\end{array}$

Tabela 3. Descrição dos eventos adversos apresentadas pelos adolescentes relacionados à vacina contra o Papillomavírus Humano quadrivalente 6, 11, 16 e 18 (recombinante). Rio de Janeiro, RJ, Brasil, 2016-2019.

\section{Evento adverso}

Dor, calor, eritema ou rubor e prurido local

Adolescentes

Alteração do nível de consciência e parestesia

Palidez, hipotensão, desmaio e síncope

Diarreia e vômitos

Vertigem e tontura

Mal-estar

Letargia

Náuseas

Diminuição da acuidade auditiva

Dispneia

Cefaleia

Fadiga

Febre de $>=39^{\circ} \mathrm{C}$ (axial)

Febre de $38,8^{\circ} \mathrm{C}$ (axial)

Mialgia

Sudorese

Indisposição

Sonolência

Tontura

Esgotamento

Dor em membros inferiores

Tremores no corpo
95,9 milhões de pessoas, representando $6,7 \%$ do total.

Em se tratando do tempo de início, entre a aplicação do imunobiológico e o começo dos sinais e sintomas, para 04 adolescentes ocorreu cerca de 30 minutos após a administração da vacina, 01 adolescente após 3 horas, 01 após 4 horas, 6 horas após a aplicação da vacina 01 adolescente e para 12 notificados o tempo de intervalo não foi informado.
Em relação ao tempo de início, a Síndrome de Reação Sistêmica Precoce (SRSP) geralmente tem o aparecimento dentro de três horas após a aplicação da vacina e apresenta de um ou mais dos seguintes sinais e sintomas: tremores, calafrios, febre, cefaleia intensa, vômitos, sonolência, prostração, cianose perioral ou de $\operatorname{dedos}^{(4)}$.

Dos eventos pós-vacinais avaliados, conforme a classificação, 01 foi classificado como Evento Adverso Grave (EAG) Segundo estudo ${ }^{(4)}$, que necessita de hospitalização de no mínimo de $24 \mathrm{~h}$ ou prolongamento, ou apresentem causa de disfunção significativa ou incapacidade persistente, causa risco morte ou morte (ex: anafilaxia), dentre outras gravidades. Este caso posteriormente foi reclassificado e encerrado como não grave, esquema mantido, pois o EAPV foi relacionado à ansiedade frente à vacinação.

Classificado como tipo de Evento Adverso Não Grave (EANG) foram encontrados 18 casos. Conforme resultados de estudo $^{(4)}$, os quais apresentaram eventos sistêmicos ou locais, moderados ou intensos, necessitando ou não de um tratamento médico ambulatorial, exames complementares e que não são encaixados nos critérios de Evento Adverso Grave.

Nesta pesquisa, os eventos adversos com maiores frequências foram relacionados a manifestações de pele/mucosa, como a palidez, manifestação cardiovascular a hipotensão e neurológica o desmaio e síncope. Tais manifestações ocorreram em 07 adolescentes.

Observou-se que 05 adolescentes manifestaram reações gastrointestinais referentes a diarreia e vômitos, 03 apresentaram eventos locais como dor, calor, eritema ou rubor e prurido local. Outras manifestações correlacionadas à cefaleia e febre (axilar) de $38,8^{\circ} \mathrm{C} \mathrm{e}>=39^{\circ} \mathrm{C}$ foram apresentadas por 03 adolescentes, vertigens e tonturas em 02 adolescentes.

As manifestações de menores frequências analisadas nesta pesquisa foram: alteração do nível de consciência, parestesia, mal-estar, letargia, náuseas, diminuição da acuidade auditiva, dispneia, fadiga, febre 
de $38,8^{\circ} \mathrm{C}$ (axial), mialgia, sudorese, indisposição, sonolência, tontura, esgotamento, dor em membros inferiores e tremores no corpo.

Achados deste estudo mostram que os eventos adversos, como a dor no local da aplicação, febre de $38^{\circ} \mathrm{C}$ ou $>=39^{\circ} \mathrm{C}$ (axial), cefaleia e síncope, são relacionadas à vacina. Em relação à febre, está associada à resposta imunológica do organismo, é frequentemente benigna e autolimitada.
A febre produz citocinas inflamatórias que atuam no hipotálamo, com liberação de prostaglandinas e elevação da temperatura sendo uma resposta fisiológica à administração de antígenos ${ }^{(4)}$.

Segundo estudo ${ }^{(4)}$, a perda da consciência também é breve, com durabilidade de menos de 1 minuto, em média de 20 a 30 segundos. $\mathrm{O}$ paciente volta ao normal em 5 ou 10 minutos. De imediato, após perder a consciência, podem ocorrer al- guns pequenos espasmos musculares nos braços, pescoço e/ou nas pernas, duram menos de 15 segundos.

A alteração do nível de consciência ocasionalmente é imediata e vem antecedida por sintomas de hipotensão arterial, sudorese fria, palidez, turvamento visual, parestesia (formigamento nos membros superiores e/ou membros inferiores) e, caso não seja adotada alguma medida, o indivíduo costuma desmaiar ${ }^{(4)}$.

Tabela 4 . Descrição dos casos com encerramentos pela da Coordenação do Programa de Imunização dos eventos adversos apresentadas pelos adolescentes Pós-vacinados pelo Papilomavírus quadrivalente 6, 11, 16 e 18 (recombinante). Rio de Janeiro, RJ, Brasil, 2016-2019.

\begin{tabular}{|c|c|c|c|c|c|}
\hline Diagnóstico & Idade & Sexo & Dose & Evolução & Encerramento pela CPI \\
\hline $\begin{array}{l}\text { Evento neurológico à } \\
\text { esclarecer }\end{array}$ & 9 & Feminino & $1^{\mathrm{a}}$ & $\begin{array}{l}\text { Cura sem } \\
\text { sequelas }\end{array}$ & Caso com encerramento, no entanto, apresentando pendências \\
\hline Síncope & 12 & Feminino & $1^{\mathrm{a}}$ & $\begin{array}{l}\text { Cura sem } \\
\text { sequelas }\end{array}$ & $\begin{array}{l}\text { Caso encerrado reclassificado como não grave. Esquema man- } \\
\text { tido, pois EAPV relacionado à ansiedade frente à vacinação }\end{array}$ \\
\hline $\begin{array}{l}\text { Erros de prescrição ou } \\
\text { indicações (fora da idade } \\
\text { recomendada) }\end{array}$ & 10 & Masculino & $1^{\mathrm{a}}$ & $\begin{array}{l}\text { Dose conside- } \\
\text { rada válida }\end{array}$ & $\begin{array}{l}\text { Solicitado ratificar as indicações da vacina HPV conforme faixas } \\
\text { etárias recomendadas pelo Programa Nacional de Imunização } \\
\text { (PNI), meninas 09 a } 14 \text { anos e meninos de } 12 / 13 \text { anos. Caso este } \\
\text { menino retorne pleiteando a D2 (06 meses depois) atender, pois } \\
\text { esquema aberto deve ser encerrado, apesar do erro cometido. }\end{array}$ \\
\hline Vômitos, diarreia e febre & 13 & Masculino & $1^{\mathrm{a}}$ & $\begin{array}{l}\text { Cura sem } \\
\text { sequelas }\end{array}$ & $\begin{array}{l}\text { Realizado o acompanhamento até a regressão dos sintomas. } \\
\text { Caso encerrado sem a necessidade de adoção de precauções } \\
\text { ou substituição do esquema. Manter calendário para idade con- } \\
\text { forme a indicação clínica e preconização do Ministério da Saúde. }\end{array}$ \\
\hline $\begin{array}{l}\text { Desmaio após forte } \\
\text { emoção }\end{array}$ & 12 & Masculino & $1^{\mathrm{a}}$ & $\begin{array}{l}\text { Cura sem } \\
\text { sequelas }\end{array}$ & $\begin{array}{l}\text { Realizado o acompanhamento com visita medica domiciliar, } \\
\text { orientada sobre os cuidados e referenciada para acompanha- } \\
\text { mento neurológico devido episódios anteriores. Caso encer- } \\
\text { rado sem a necessidade de adoção de precauções ou substi- } \\
\text { tuição do esquema. Manter calendário para idade conforme a } \\
\text { indicação clínica e preconização do Ministério da Saúde. }\end{array}$ \\
\hline Vômitos e vertigem & 13 & Feminino & $1^{\mathrm{a}}$ & $\begin{array}{l}\text { Cura sem } \\
\text { sequelas }\end{array}$ & $\begin{array}{l}\text { Realizado o acompanhamento por } 4 \text { semanas, mas houve } \\
\text { melhoras logo após o único episódio de vômito. Caso encer- } \\
\text { rado sem a necessidade de adoção de precauções ou substi- } \\
\text { tuição do esquema. Manter calendário para idade conforme a } \\
\text { indicação clínica e preconização do Ministério da Saúde. }\end{array}$ \\
\hline $\begin{array}{l}\text { Tontura, vertigem, sono- } \\
\text { lência, mal estar geral e } \\
\text { indisposição }\end{array}$ & 13 & Masculino & $1^{\mathrm{a}}$ & $\begin{array}{l}\text { Cura sem } \\
\text { sequelas }\end{array}$ & $\begin{array}{l}\text { Realizado o acompanhamento por } 4 \text { semanas. Caso encer- } \\
\text { rado sem a necessidade de adoção de precauções ou substi- } \\
\text { tuição do esquema. Manter calendário para idade conforme a } \\
\text { indicação clínica e preconização do MS. }\end{array}$ \\
\hline $\begin{array}{l}\text { Erros de prescrição ou } \\
\text { indicações (fora da idade } \\
\text { recomendada) }\end{array}$ & $\begin{array}{c}17 \text { e } \\
18\end{array}$ & Masculino & $\begin{array}{l}1^{\mathrm{a}} \mathrm{e} \\
2^{\mathrm{a}}\end{array}$ & $\begin{array}{l}\text { Doses con- } \\
\text { sideradas } \\
\text { válidas }\end{array}$ & $\begin{array}{l}\text { Reforçamos sobre a necessidade de avaliar a caderneta va- } \\
\text { cinal e conferindo idade correta, para decidir sobre a vacina a } \\
\text { ser aplicada, ler atentamente os frascos dos imunobiológicos } \\
\text { antes da inoculação, sem esse procedimento estaremos fragi- } \\
\text { lizados, sob risco de cometer novos erros de imunização. Hou- } \\
\text { ve dois erros na } 1^{\text {a }} \text { e } 2^{\text {a }} \text { doses fora da faixa etária preconizada. } \\
\text { Validar as doses aplicada, oportunizar imunização segura com } \\
\text { o calendário preconizado para idade. }\end{array}$ \\
\hline
\end{tabular}




\section{artigo}

Bernado da Silva, M.R.; Conceição, A.S.F.; Magno da Silva, D.; Ramado, A.D.A.; Andrade, J.G.; Reis T.L.; Souza, R.S.;

Eventos adversos pós-vacinais papilomavírus humano em adolescentes

Erros de prescrição ou indicações (fora da idade recomendada)
9

Feminino

2

Dor no local da injeção e

sonolência

Vômitos

Lipotímia

Cefaléia, palidez, fadiga

intensa e esgotamento

Síncope

Dor muscular, dor no local da Injeção, cefaleia e febre

12

12

Masculino

4

Masculino

10

Feminino
Masculino $1^{\mathrm{a}}$

13 Masculino $1^{\mathrm{a}}$

Cura sem sequelas

Cura sem sequelas

11 Masculino $1^{\text {a }} \quad$ sequelas

$1^{a}$

$1^{\mathrm{a}}$

$1^{\mathrm{a}}$

Cura sem sequelas

Cura sem sequelas

Cura sem sequelas

Cura sem sequelas

\section{Síncope}

Feminino

$1^{\mathrm{a}}$

Cefaléia e náuseas

\section{1}

Masculino

Prurido, eritema, dor no local da injeção e calor no local da injeção
Realizado D1 em 08/11/16 e D2 em 29/03/17, ou seja, administração da vacina com intervalo de aproximadamente 3 meses e 15 dias entre as doses, sendo o preconizado 6 meses. Reforçamos sobre a necessidade de avaliar a idade do paciente, e doses já aplicadas com seus respectivos intervalos e conferindo dados fundamentais para uma imunização segura. Sem esse procedimento estaremos fragilizados, sob risco de cometer novos erros de imunização. Validar as doses aplicadas. Caso encerrado com necessidade de completar esquema HPV e as demais vacinas do calendário vacinal para idade.

Realizado acompanhamento até regressão dos sintomas, com uso de compressas frias e uso de antitérmico profilático, prescrito pelo profissional médico. Caso encerrado cura sem sequela, com adoções de precaução.

Caso encerrado sem a necessidade de determinação de conduta específica ou orientação. EAPV já descrito em literatura (Manual de Vigilância de EAPV - MS) para os imunobiológicos em questão.

Mantido esquema, considerando precaução em Atenção Primária como realizar acolhimento, fornecendo ambiente privativo e confortável para reduzir algum "stress" antes da vacinação. Vacinação do adolescente sentado, realizar monitoramento do mesmo por 1 hora após a vacinação e liberar para domicilio com orientações, após certificar que está clinicamente bem.

Caso encerrado após remissão completa das manifestações clínicas. Esquema de HPV mantido, vacina da Febre Amarela e Meningocócica C encerrados.

Caso encerrado com síncope, relacionada à ansiedade pela vacinação. Esquema HPV mantido.

Orientamos vacinar com precauções no CRIE. Agendar. Aguardamos

Caso encerrado com cura sem sequelas. Recomenda-se dar continuidade ao esquema vacinal de rotina de acordo com o calendário vigente e intervalos preconizados pelo Programa Nacional de Imunizações e de acordo com a faixa etária da usuária. Conforme recomendação técnica, vacinar a adolescente sentada e orientar que a mesma aguarde cerca de 15 a 30 minutos após a vacinação. Tendo como objetivo avaliar a possível ocorrência de Eventos Adversos imediatos. Orientar o usuário/responsável quanto à importância do retorno à Unidade em caso de ocorrência de reações pós-vacinais visando a notificação e o acompanhamento clínico até a melhora do quadro.

Caso encerrado com cura sem sequelas. Recomenda-se se manter o esquema sequencial conforme calendário vacinal vigente do Programa Nacional de Imunizações. Reforçar a orientação ao responsável pela adolescente quanto à importância do retorno à Unidade em caso de ocorrência de EAPV, após a completude do esquema com a $2^{\circ}$ dose de HPV.

Após a remissão completa das manifestações clínicas e sem relato de agravamento do quadro, o caso pode ser encerrado com o esquema vacinal mantido. Enfatizamos a importância da orientação sobre a aplicação de compressas frias intermitentes nos casos de reação local. 
Cefaléia, síncope vago-vagal e reação febril

Masculino $\quad 1^{\text {a }}$

Cura sem sequelas

Sudorese excessiva ou hiperidrose, vômitos e palidez

11 Masculino $1^{\text {a }}$

Cura sem sequelas

\section{Cefaleia e dispneia} 14

14

Masculino

$1^{\mathrm{a}}$

\section{sequelas}

Cura sem

Febre
9
Feminino
Caso encerrado com cura sem sequelas. Recomenda-se a continuidade do esquema sequencial de HPV e Febre Amarela conforme calendário vigente e os intervalos entre as doses preconizados pelo Programa Nacional de Imunizações, considerando a faixa etária do usuário. Sendo este um adolescente com condições específicas de saúde e com predisposição a apresentar reações de ansiedade, reforçamos a orientação de vaciná-lo sempre sentado, orientar a equipe técnica para que realize a observação clínica deste usuário durante no mínimo 40-60 min dentro da Unidade de Atenção Primária após a aplicação de vacinas, tendo como objetivo avaliar precocemente a possível ocorrência de Eventos Adversos. Orientar o responsável quanto à importância do retorno à Unidade em caso de ocorrência de reações pós-vacinais visando a notificação e o acompanhamento clínico até a melhora do quadro.

Caso encerrado com cura sem sequelas. Reações compatíveis com quadro de ansiedade relacionado à vacinação. Recomenda-se a continuidade do esquema sequencial conforme calendário vacinal vigente, considerando a faixa etária do adolescente e os intervalos entre as doses recomendados pelo Programa Nacional de Imunizações. Este adolescente deverá receber vacinas de rotina na posição sentado e deverá ser observado clinicamente dentro da Unidade por no mínimo 20 minutos após a administração de imunobiológicos tendo como objetivo a identificação precoce de possivel EAPV. Em caso de novo EAPV, o tratamento deverá ser sintomático.

Com encerramento, no entanto no sistema não há justificativas / recomendações.

Caso encerrado com cura sem sequelas. Recomenda-se manter esquema de HPV quadrivalente, conforme calendário vacinal vigente do Programa Nacional de Imunizações e considerando a faixa etária do usuário. Em caso de febre no período pós-vacinal, utilizar antitérmico de acordo com a conduta médica. Reforçar a orientação junto ao responsável quanto à importância do retorno à Unidade em caso de novas reações.
Com relação ao parecer da Coordenação do Programa de Imunizações, dos 22 casos estudados, 01 apresenta diagnóstico neurológico a esclarecer, inclassificável, com evolução para cura sem sequelas e encerramento com pendências. $\mathrm{O}$ caso foi encerrado como pendente pela CPI, carente de detalhes, necessitando de um laudo ou parecer do neurologista. Adolescente com diagnóstico de epilepsia na infância, sem alterações do quadro neurológico e a mãe não desejou realizar a continuidade do esquema da vacina contra o Papillomavírus Humano.

Os 3 Erros de Imunização (EI) apresentaram erros de prescrição ou indicações fora da idade recomendada. Profissionais receberam orientações da
Coordenção da necessidade de avaliar a caderneta vacinal, conferindo idade correta, para decidir sobre a vacina a ser aplicada. Avaliar as doses já aplicadas com seus respectivos intervalos e conferindo dados fundamentais para uma imunização segura. Ratificar as indicações da vacina HPV conforme faixas etárias recomendadas pelo Programa Nacional de Imunizações (meninas 09 a 14 anos e meninos de 12 e 13 anos). Sem esses procedimentos torna-se frágil a avaliação, sob risco de cometer novos erros de imunização.

Os diagnósticos de vômitos, diarreia, febre, reação febril, vertigem, sonolência, mal estar geral, cefaleia, náuseas, palidez, fadiga intensa, esgotamento, indisposi- ção com evolução para cura sem sequelas, foram realizados acompanhamentos até a regressão dos sintomas, casos encerrados com parecer da Coordenação sem a necessidade de adoção de precauções ou substituição do esquema. Mantido o calendário para idade conforme a indicação clínica e preconização do Ministério da Saúde, EAPVs já descritos em literatura (Manual de Vigilância de EAPV - MS) para os imunobiológicos em questão. Em caso de febre no período pós-vacinal, utilizar antitérmico de acordo com a conduta médica e reforçar a orientação junto ao responsável quanto a importância do retorno à unidade em caso de novas reações.

Relacionados às manifestações locais, 
os diagnósticos, como dor e calor no local da injeção, prurido e eritema, após a regressão dos sinais e sintomas, sem relatos de agravamento do quadro e acompanhamento por 30 dias, com encerramento de cura sem sequelas, as justificativas e recomendações da Coordenação foram de uso de antitérmico profilático, prescrito pelo profissional médico. Enfatizada a importância da orientação sobre a aplicação de compressas frias intermitentes nos casos de reação local. O diagnóstico dor muscular, dor no local da injeção, cefaleia e febre ocorreu em 01 caso, este, com parecer da Coordenação com orientação de vacinar com precauções no CRIE. Registro de 01 caso com diagnóstico de cefaleia e dispneia com cura sem sequela, com encerramento, no entanto, no sistema não há justificativas e recomendações.

Os casos com diagnóstico de síncope e desmaio após forte emoção tiveram encerramento com cura sem sequelas. Reações compatíveis com quadro de ansiedade relacionado à vacinação. A Coordenação do Programa de Imunização recomenda a continuidade do esquema sequencial conforme calendário vacinal vigente, considerando a faixa etária do adolescente e os intervalos entre as doses recomendados pelo PNI. Estes adolescentes deverão receber vacinas de rotina na posição sentada e deverão ser observados clinicamente dentro da unidade por no mínimo 20 minutos após a administração de imunobiológicos, tendo como objetivo a identificação precoce de possível EAPV. Em caso de novo EAPV, o tratamento deverá ser sintomático e orientar o usuário/responsável quanto à importância do retorno à unidade em caso de ocorrência de reações pós-vacinais visando a notificação e o acompanhamento clínico até a melhora do quadro.

Conforme estudo ${ }^{(4)}$, os eventos relacionados à síncope vasovagal podem ocorrer após qualquer vacinação, é muito frequente em adolescentes e adultos jovens, sendo comum em pessoas com instabilidade afetiva. Alguns fatores aumentam a probabilidade de ocorrer a síncope, como: jejum, aposentos quen-
Os casos com diagnóstico de síncope e desmaio após forte emoção tiveram en-

cerramento com cura

sem sequelas. Rea-

ções compatíveis com

quadro de ansiedade

relacionado à vacina-

ção. A Coordenação

do Programa de Imu-

nização recomenda

a continuidade do

esquema sequencial

conforme calendá-

rio vacinal vigente,

considerando a faixa

etária do adolescente

e os intervalos entre

as doses recomenda-

dos pelo PNI. tes e superlotados, ficar de pé por muito tempo e fadiga. O recomendado é a vacinação do adolescente sentado e após a administração da vacina, aguardar no mínimo 15 minutos para que se observe alguma reação.

Para estar relacionada a um procedimento de vacinação, a dor deverá ocorrer no momento da administração ou logo após. Ela é uma experiência pessoal cuja medida depende da resposta subjetiva da pessoa que a experimenta e a expectativa de dor ou um choque emocional súbito. De todas as reações locais, a dor é o maior desafio e difícil de descrever, quantificar e padronizar ${ }^{(4)}$.

De acordo com a pesquisa, as manifestações clínicas sistêmicas neurológicas apresentaram-se na sua maioria como síncope vasovagal, mais frequente em adolescentes e adultos jovens após algum estímulo desencadeante como dor intensa, expectativa de dor ou um choque emocional súbito. Os eventos locais, gastrointestinais, pele/mucosa, cardio vasculares e outras manifestações, estão relacionados à vacina.

Neste estudo, 13 casos de eventos adversos estão relacionados à vacinação do HPV realizada simultaneamente com outros imunobiológicos. Concomitantemente com a vacina Papilomavírus Humano, foram administradas a Meningocócica Conjugada C, Febre Amarela e Influenza. Os principais eventos adversos associados às vacinas administradas simultaneamente com a do HPV, em relação à manifestação local de dor, nas vacinas de HPV e Febre Amarela é esperado ${ }^{(1)}$.

As manifestações gerais, como febre e cefaleia, de acordo com o Ministério da Saúde ${ }^{(1)}$, são descritas em literatura para os imunobiológicos HPV, Febre Amarela e Meningocócica C. Classificados como anafilaxias, os Eventos Adversos como hipotensão e parestesia foram relacionados com as vacinas do HPV, Febre Amarela e Meningocócica C.

\section{CONCLUSÃO}

A pesquisa permitiu um amplo conhecimento acerca do tema. Com base nos 
estudos realizados, se demonstrou que os eventos adversos com maiores frequências apresentaram evolução para cura sem sequelas, relacionados à: manifestações de pele/mucosa como a palidez, manifestação cardiovascular a hipotensão e neurológica o desmaio e síncope.

O levantamento realizado afirma que a maioria dos eventos adversos pós-vacinais nos adolescentes estão relacionados à ansiedade e medo frente à vacinação, com ausência de ocorrência de eventos adversos que sejam considerados graves, tanto que das 22 fichas de notificações analisadas, sendo 19 de eventos adversos pós-vacinais e 03 Erros de Imunização (EI), somente 01 foi classificada como grave e, este, posteriormente, foi reclassificado e encerrado como não grave.

Sugere-se melhora na qualificação das fichas de eventos adversos analisadas nesta pesquisa. Apresentaram-se em sua maioria, frágeis sem muitas informações detalhadas dos sinais e sintomas e do monitoramento do quadro clínico dos adolescentes. Além disso, também vale ressaltar a necessidade de sensibilizar os profissionais que atuam nas salas de vacinas, da importância da notificação em tempo oportuno, das boas práticas em imunizações, assegurando uma imunização segura, eficaz e evitando ruídos negativos e inadequados da vacinação. Afim de que, a população tenha confiabilidade na qualidade e segurança da vacina contra o HPV.

Após a análise, aponta-se o pequeno número de notificações inseridas no Sistema em um período de 2 anos e 7 meses. No entanto, não é possível afirmar a subnotificação dos eventos adversos pela coleta de dados usados para desenvolver o trabalho em questão.

Diante do exposto, conclui-se que, mesmo diante da ocorrência de tais eventos adversos, é relevante e vantajosa como estratégia de prevenção dessa infecção viral e do câncer do colo uterino, a realização do esquema vacinal completo. E que a vacina quadrivalente Papilomavírus 6, 11, 16 e 18 (Recombinante) apresenta boa tolerabilidade e é segura.

\section{AGRADECIMENTO}

Agradecimento especial à MSc Isabela dos Santos Souza Diretora da DVS DA CAP 5.1 que gentilemente colaborou com a nossa pesquisa.

\section{REFERÊNCIAS}

1. Ministério da Saúde, Instituto Nacional do Câncer (BR). Estimativa 2018. Incidência do Câncer no Brasil [Internet]. Rio de Janeiro: INCA, 2017 [acesso em 21 jan 2019]. Disponivel em: http://www. inca.gov.br/estimativa/2018/estimativa-2018.pdf.

2. International Agency of Research on Cancer (IARC). Working group on the evaluation of carcinogenic risks to humans: Human papillomaviruses. Monographs on the evaluation of carcinogenic risks to humans [Internet]. 2007 [acesso em 28 abr 2019]. 90:1636. Disponivel em: https://monographs.iarc.fr/wp-content/ uploads/2018/06/mono90.pdf.

3. Instituto Nacional de Câncer José Alencar Gomes da Silva (INCA). Estimativa 2018: Incidência de Câncer no Brasil [Internet]. Rio de Janeiro: INCA, 2018 [acesso em 23 abr 2019]. Disponivel em: http://www1.inca.gov.br/estimativa/2018/.

4. Ministério da Saúde, Secretaria de Vigilância em Saúde, Departamento de Vigilância das Doenças Transmissiveis (BR). Manual de vigilância epidemiológica de eventos adversos pós-vacinação / Ministério da Saúde, Secretaria de Vigilância em Saúde, Departamento de Vigilância das Doenças Transmissíveis. - 3. ed. - Brasília: Ministério da Saúde, 2014.250 p.

5. Junior JBS. 40 anos do Programa Nacional de Imunizações: uma conquista da Saúde Pública brasileira. Epidemiol. Serv. Saúde [Internet]. 2013 mar. [acesso em 27 mai 2019]; 22(1). Disponivel em: http://scielo.iec.gov.br/scielo.php?script=sci_arttext\&pid=S1679-49742013000100001.

6. Araujo TME, Carvalho PMG, Vieira RDF. Eventos adversos pós-vacinais ocorridos em Teresina. Revista brasileira de enfermagem [Internet]. 2007 Aug [acesso em 27 mai 2019]; 60(4):444-448. Disponível em: http://www.scielo.br/scielo.
php?script=sci_arttext\&pid=S003471672007000400016\&lng=en\&nrm=iso.

7. Santos MCS, Netto VBP, Andrade MS. Prevalência e fatores associados à ocorrência de eventos adversos pós-vacinação em crianças. Acta Paul Enferm. 2016; 29(6):626-32

8. Bisetto LHL, Ciosak SL. Análise da ocorrência de evento adverso pós-vacinação decorrente de erro de imunização. Revista Brasileira de Enfermagem [Internet]. 2017 Feb [acesso em 27 mai 2019]; 70(1):87-95. Disponivel em: http://www.scielo.br/scielo. php?script=sci_arttext\&pid=S003471672017000100087\&Ing=en\&nrm=iso.

9. Ballalai I, Bravo F (Org). Imunização: Tudo o que você sempre quis saber. Rio de Janeiro: RMCOM; 2016. p 97.

10. Gil AC. Método e Técnicas de Pesquisa Social. 5. Ed. São Paulo: Ed. Atlas; 1995.

11. Oliveira MF. Metodologia científica: um manual para a realização de pesquisas em Administração/ Maxwell Ferreira de Oliveira. Catalão: UFG; 2011. 72 p.: il.

12. Malhotra N. Pesquisa de marketing. 3.ed. Porto Alegre: Bookman; 2001.

13. Moresi E. Metodologia da Pesquisa. Universidade Católica de Brasília - UCB, Pró-reitoria de pós-graduação - PRPG, Programa de pós-graduação stricto sensu em gestão do conhecimento e tecnologia da informação. Brasília - DF Mar 2003.

14. Bordalo AA. Estudo transversal e/ou longitudinal. Rev. Para. Med. [Internet]. 2006 dez [acesso em 27 mai 2019]; 20(4). Disponivel em: http://scielo.iec.gov.br/scielo.php?script=sci_arttext\&pid=S0101-59072006000400001. 